\title{
Obliteración del seno frontal para el tratamiento de fístulas de LCR y meningoencefaloceles
}

\author{
Frontal sinus obliteration for treatment of CSF leaks and \\ meningoencephaloceles
}

María P lisbona A¹, Asís Lorente M², Rafael Fernández L', Luis González M², Juan Alberdi V².

\section{RESUMEN}

El tratamiento endoscópico de las fístulas de LCR se ha convertido en el gold standard. No obstante, aquellas localizadas en seno frontal presentan serias dificultades para su abordaje endoscópico, especialmente si se asocia la existencia de un meningoencefalocele. En estos casos se debe valorar el abordaje externo con obliteración del seno frontal. Presentamos dos casos de fístulas de LCR cuyo origen fue debido a sendos meningoencefaloceles protruyendo a través de la pared posterior del seno frontal y que precisaron de un abordaje externo para su tratamiento.

Palabras clave: Seno frontal, meningoencefalocele, obliteración.

\begin{abstract}
Endoscopic treatment of CSF leak is the Gold Standard. Nevertheless, at frontal sinus it is very difficult the endoscopic approach, especially if there is a meningoencephalocele associated. In these cases, an external approach with frontal sinus obliteration should be considered. We present two cases of CSF leaks whose origin are meningoencephaloceles that protrude from posterior frontal sinus wall, treated with an external approach.
\end{abstract}

Key words: Frontal sinus, meningoencephalocele, obliteration.

${ }^{1}$ Médico, Servicio de Otorrinolaringología, Hospital Universitario Miguel Servet, Zaragoza, España.

${ }^{2}$ Médico, Servicio de Neurocirugía, Hospital Universitario Miguel Servet, Zaragoza, España. 


\section{INTRODUCCIÓN}

Las fístulas de LCR se producen habitualmente a nivel de la lámina cribosa, a nivel etmoidal, en el seno esfenoidal y, de forma menos frecuente, en el seno frontal por ser las zonas más frágiles de la base del cráne ${ }^{1}$. El tratamiento endoscópico se considera actualmente de elección por su menor morbilidad. Sin embargo, acceder al seno frontal en su totalidad puede presentar serias dificultades. Particularmente es complejo el acceso a la pared posterior del seno frontal para la reparación de un meningoencefalocele y de la fístula de LCR asociada. En estos casos el abordaje externo para realizar la reconstrucción dural y la obliteración del seno frontal es una alternativa al abordaje endoscópico, siendo según algunos autores la técnica a realizar².

\section{CASO CLÍNICO}

Caso 1. Varón de 28 años que ingresa por meningitis neumocócica. Hace 15 años, había sufrido un traumatismo cráneo-encefálico frontal. La gammagrafía muestra captación en el trayecto fronto-etmoidal, compatible con una fístula de LCR. En la tomografía computarizada (TC) se observa ocupación del seno frontal derecho y un pequeño defecto óseo en la pared posterior. La RNM muestra una zona de encefalomalacia a nivel ántero-inferior del lóbulo frontal derecho, que parece extenderse e introducirse hacia el seno frontal (Figuras 1A y 1B). Ante estos hallazgos se decide realizar una intervención quirúrgica, planteando inicialmente un abordaje endoscópico. Se realiza una etmoidectomía anterior y se llega hasta el ostium del seno frontal, comprobándose que no se puede acceder a la zona posterolateral del seno. Se decide efectuar, mediante una incisión coronal, una osteoplastía externa del seno con colgajo pediculado de periostio. Para marcar los límites del seno, al paciente previamente se le había realizado una radiografía de senos paranasales en escala 1:1, sin magnificación. Esta radiografía se esterilizó y, tras disecar las partes blandas, se superpuso la placa en el hueso para delimitar de una forma bastante precisa los límites del seno. La apertura del seno se realizó con una sierra en una dirección angulada, no de forma perpendicular. Tras la apertura del seno, se observa el meningoencefalocele protuyendo a través de un defecto de la pared posterior (Figura 2A). Se reseca completamente la mucosa del seno, incluyendo la del infundíbulo, mediante fresado. Después se coagula con bipolar el meningoencefalocele para retraerlo y se coloca una plastía con dura artificial; a continuación se aplica un biosellante, y luego, un osteoinductor. Tras pedicular el colgajo de periostio, se rellena el seno con grasa abdominal autóloga reponiéndose el colgajo óseo con miniplacas (Figura 2B).

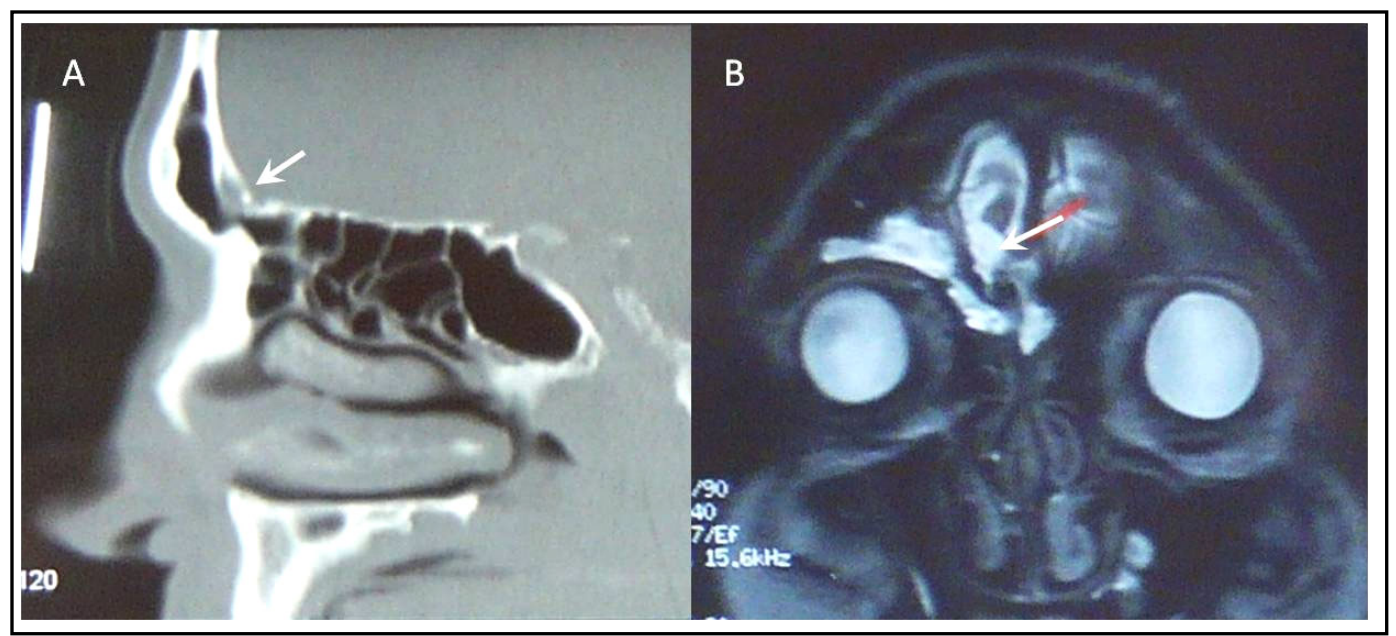

Figura $1 \mathrm{~A}$. TC sagital observándose la protrusión del meningocele en seno frontal a través de la tabla interna.

Figura 1B. RNM T2 coronal muestra la ocupación del seno frontal con LCR y la protrusión del meningoencefalocele. 


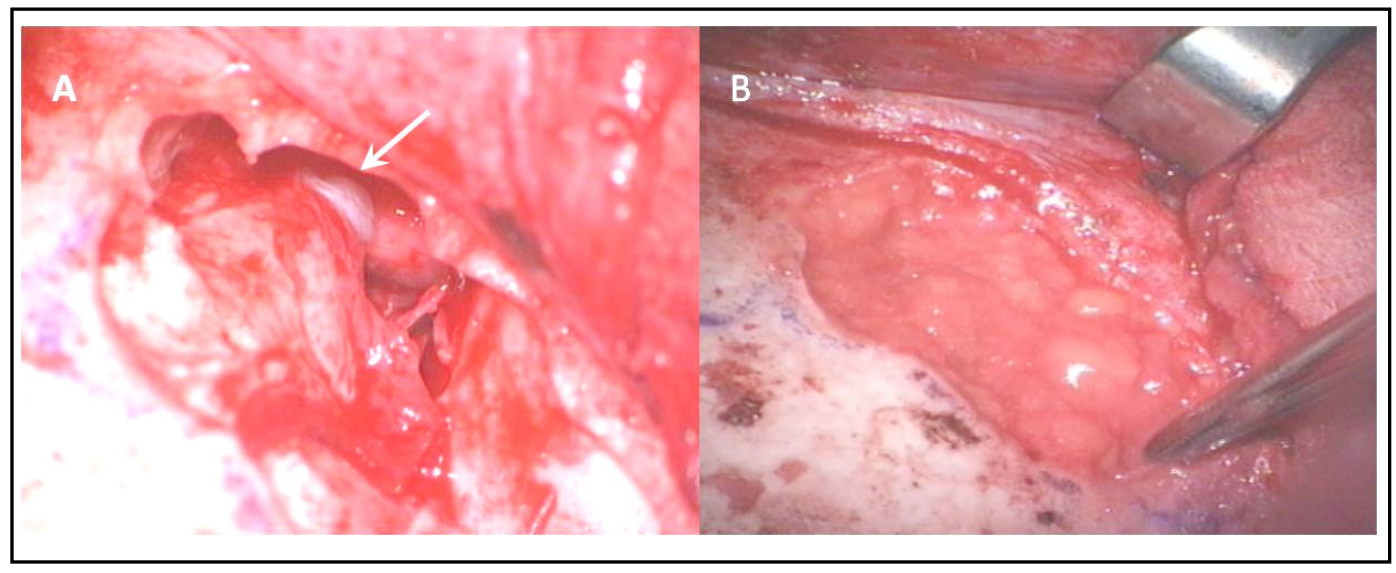

Figura 2A. Imagen del meningoencefalocele tras la apertura del seno frontal. Figura 2B. Rebatimiento del colgajo de periostio y obliteración con grasa.

Caso 2. Varón de 66 años, que refería rinorrea previa y que ingresa por meningitis neumocócica. La TC muestra ocupación del seno frontal izquierdo con dehiscencia en la zona meatal supraorbitaria (Figura 3A). La RNM muestra imagen sospechosa de meningoencefalocele. Por su aparente localización medial, se decide realizar reparación endoscópica, técnica con la que no se logra acceder bien a la zona. Posteriormente con la ayuda del neuronavegador (StealthStation ${ }^{\circledR}$ TREON $^{\mathrm{TM}}$ Medtronic) se realiza un abordaje externo supraciliar con colgajo osteoplástico, efectuándose la incisión en una arruga de expresión. En este caso se usó el neuronavegador, utilizando una secuencia de TC para delimitar con precisión los límites del colgajo. La apertura se realiza con una sierra manteniendo igualmente una angulación aguda respecto a la calota. Se visualiza el meningoencefalocele (Figura 3B), que se coagula, y se procede a obliterar el seno frontal con grasa, con la misma técnica que en el caso anterior.

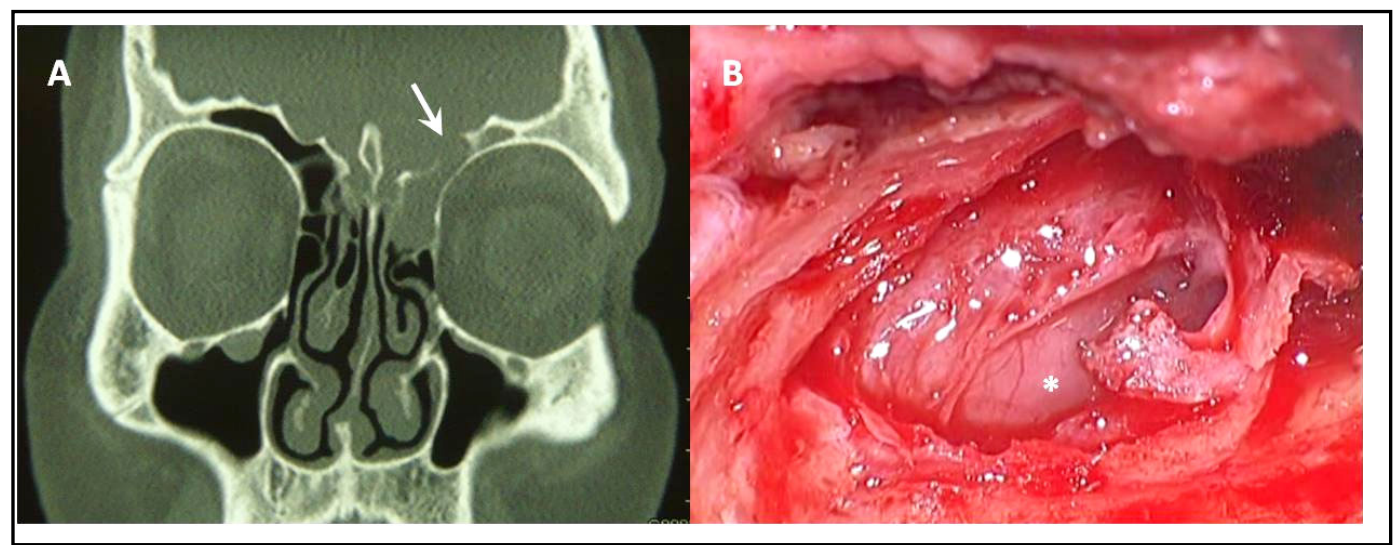

Figura 3A. TC coronal muestra el defecto óseo (flecha) y la ocupación del seno frontal.

Figura 3B. Imagen del meningoencefalocele, apreciándose tejido cerebral en la parte inferior. 
En ambos casos se colocó un drenaje lumbar externo, que se mantuvo durante 5 días.

Tras un seguimiento de 15 y 20 meses ambos pacientes presentan un resultado satisfactorio, sin recidiva de la fístula y sin complicaciones en forma de mucocele 0 algias faciales. Las TC de control muestran una correcta obliteración (Figuras 4A y 4B).

\section{DISCUSIÓN}

En 1951 Wigand publica la primera reparación endoscópica de una fístula de LCR ${ }^{1}$. Desde entonces el tratamiento de esta patología ha evolucionado paralelamente al desarrollo de las técnicas endoscópicas, siendo ésta la técnica de elección. Sin embargo, las fístulas del seno frontal son un subgrupo difícil de tratar, ya que habitualmente se deben a un defecto en la pared posterior del seno. Además, la existencia de un meningoencefalocele hace técnicamente más difícil la reparación endoscópica. Hay que tener en cuenta que hasta el $27 \%$ de las fístulas de LCR traumáticas pueden tener asociado un meningoencefalocele, llegando esta cifra hasta $91 \%$ en el caso de las espontáneas ${ }^{3}$. Se ha planteado que si el origen de la fístula se encuentra lateral al nervio supraorbitario, o si está en la zona superior, el abordaje externo puede ser más adecuado².

La obliteración del seno frontal, popularizada por Goodale y Montgomery en los años ‘ 50 , es un tratamiento generalmente bien tolerado $0^{4}$.

Las dos incisiones más utilizadas son la coronal y la supraciliar. La coronal proporciona un mejor acceso al seno y no ocasiona un defecto estético si se puede hacer posterior a la línea del cabello. Al estar la incisión alejada de la zona de apertura ósea, ofrece mejores resultados de cicatrización en el caso de existir fístula de LCR, infección, o si la zona ha sido irradiada ${ }^{4,5}$. Con la incisión supraciliar se corre el riesgo de seccionar los nervios supratroclear y supraorbitario, generando insensibilidad en la zona frontal, además del defecto estético que se genera, lo que puede ser válido en pacientes mayores o con alopecía.

El neuronavegador, si bien aporta una mayor precisión a la hora de planificar la cirugía, no es imprescindible. En casos de senos muy pequeños

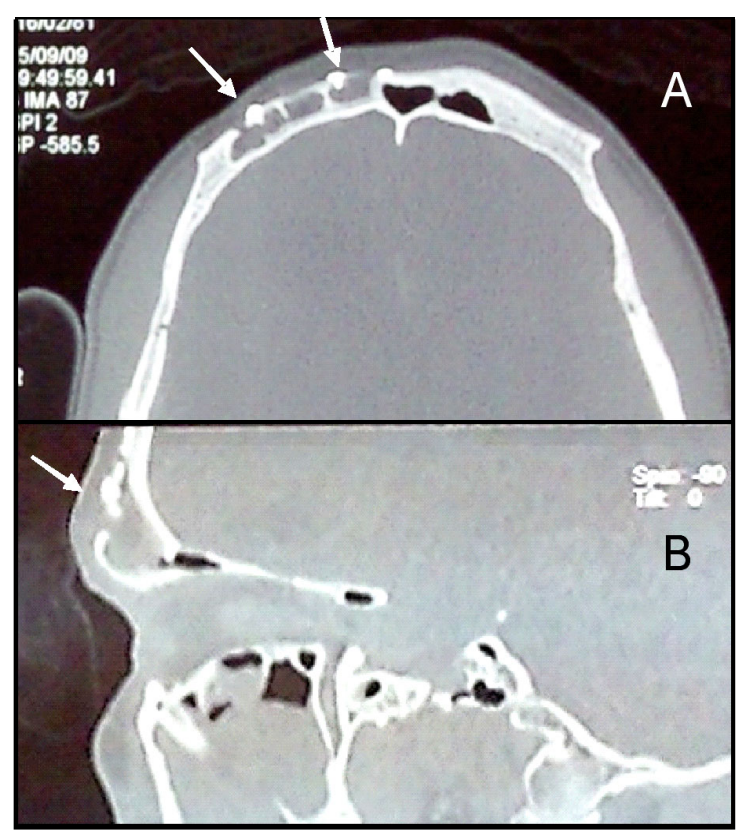

Figura 4A. TC axial de control (caso 1) mostrando la obliteración del seno frontal (flechas señalan el material de osteosíntesis).

Figura 4B. TC sagital (caso 1): se observa la obliteración total del seno.

puede resultar de especial utilidad. El aumento del tiempo quirúrgico por tener que realizar la navegación es escaso, aproximadamente unos 10 minutos, si bien genera un mayor coste.

Para realizar la apertura del seno se debe angular la sierra de tal forma que la tabla externa quede con una mayor superficie que la interna, evitándose así el hundimiento del colgajo ${ }^{4}$. Es fundamental realizar la escisión de la mucosa en su totalidad ${ }^{4,5}$ para evitar que se generen mucoceles; ${ }^{6}$ esto en algunas series llega al $10 \%$, si bien no todos son sintomáticos ${ }^{7}$.

Habitualmente los meningoencefaloceles no son de gran tamaño, y se considera que el contenido herniario está desvitalizado y no es funciona ${ }^{8,9}$, por lo que se recomienda su coagulación y retracción 0 exéresis extradural. Únicamente en herniaciones grandes se debería plantear la reintroducción.

No existe consenso en la literatura respecto a la colocación de un drenaje lumbar externo $0^{1,6,10,11,13}$. Drenando LCR se consigue disminuir la presión sobre la zona reconstruida, facilitando la cicatrización del tejido; no obstante, algunos autores recomiendan su uso sólo en casos de fístulas asociadas a hipertensión intracraneal10,11. 
Al realizar un colgajo osteoplástico con conservación del periostio de la tabla externa se reduce la posibilidad de una osteomielitis, ${ }^{2,12}$. A nivel global, el $92 \%$ de las complicaciones son menores y no precisan de un tratamiento agresivo. La más frecuente es la existencia de dolor facial, el que suele ser transitorio².

Respecto al material empleado para la obliteración, el más común es la grasa del propio paciente. Aunque se ha comprobado que el volumen de grasa disminuye hasta $20 \%$ a los 15 meses, ésta sería reemplazada por tejido fibroso u óse $0^{2}$. Las complicaciones del sitio de la extracción son mínimas y autolimitadas ${ }^{12}$ y no suelen generar problemas estéticos a nivel frontal. Como alternativa a la grasa se pueden usar cementos artificiales, como la hidroxiapatita 0 apatita carbonatada ${ }^{14-16}$. Estos materiales presentan la ventaja de no disminuir de volumen ${ }^{4,15}$ y de evitar la morbilidad del sitio de extracción. El riesgo de infección es variable, oscilando entre $5 \%$ y $13 \% \%^{14-16}$, y puede ser necesaria la reintervención para retirar el material y tratar la infección ${ }^{16}$. Además, conlleva un importante mayor coste. La tasa de fracaso global se estima entre $0 \%$ y $10 \%$.

Como alternativa, recientemente se ha descrito la técnica endoscópica modificada de Lothrop para reparar fístulas en zonas poco accesibles del seno frontal, o para cirugía de revisión en una obliteración fallida ${ }^{13,17}$. Descrita en 1914, la técnica original consistía en combinar un abordaje externo y uno transnasal para realizar una resección del suelo medial del seno frontal, del tabique nasal en su porción superior y del septo intersinusal con el fin de conseguir un buen drenaje del seno frontal13,18. A finales de la década de los ' 90 se publicaron varios trabajos que describían una variante de esta técnica realizada por endoscopía, especialmente para el tratamiento de sinusitis crónicas, consiguiendo un amplio drenaje del seno frontal, con buenos resultados, comparables a la técnica de la obliteración con grasa ${ }^{19,20}$.

Mediante esta modificación algunos autores reportan el tratamiento exitoso de fístulas de LCR con su origen en la pared posterior del seno frontal ${ }^{13}$, siendo clave realizar una amplia resección del suelo del seno. Sin embargo, el acceso a la zona más lateral y a la superior, continuaría siendo difícil por el acuñamiento que genera la forma convexa de la pared posterior del seno frontal ${ }^{13}$.

\section{CONCLUSIONES}

El abordaje externo proporciona un excelente acceso a la totalidad del seno frontal para poder realizar con éxito la reconstrucción de la pared posterior en casos de meningoencefaloceles y la obliteración del seno.

La obliteración con grasa proporciona buenos resultados en el tratamiento de fístulas de LCR, siendo esta técnica bien tolerada.

La resección y fresado de la totalidad de la mucosa del seno es esencial para evitar la formación de mucoceles, que pueden generar un importante malestar en el paciente obligando, incluso, a realizar una cirugía de revisión.

Es recomendable el uso del navegador para marcar los límites de la zona de apertura del seno y no invadir la cavidad craneal, y también en el caso de usar una incisión supraciliar para minimizar el defecto estético.

\section{BIBLIOGRAFÍA}

1. Schlosser R, Bolger W. Nasal cerebrospinal fluid leaks: Critical review and surgical considerations. Laryngoscope 2004; 114: 255-65.

2. Soyka M, Annen A, Holzmann D. Where endoscopy fails: Indications and experience with the frontal sinus fat obliteration. Rhinology 2009; 47: 136-40.

3. Banks CA, Palmer JN, Chiu Ag, O'Malley BW Jr, WoodWorth BA, Kennedy DW. Endoscopic closure of CSF rhinorrhea: 193 cases over 21 years. Otolaryngol Head Neck Surg 2009; 140(6): 826-33.

4. López Llanes A, Llorente Pendás J, Suárez Fente V, Burón Martínez G, Suárez Nieto C. Osteoplastia frontal: nuestra experiencia. Acta Otorrinolaringol Esp 2003; 54: 429-34.

5. Bertrán JM, Pérez C, Martínez A. Osteoplastia frontal: Estudio de 47 casos. Acta Otorrinolaringol Esp 1998; 49: 380-4.

6. Mirza S, Thaper A, McClelland L, Jones NS. Sinonasal cerebrospinal fluid leaks: management of 97 patients over 10 years. Laryngoscope 2005; 115(10): 1774-7.

7. Weber R, Draf W, Keerl R, Kahle G, Schinzel $S$, Thomann S, Lawson W. Osteoplastic frontal sinus 
surgery with fat obliteration: technique and long-term results using magnetic resonance imaging in 82 operations. Laryngoscope 2000; 110(6): 1037-44.

8. Pappas DG, Pappas DG, Hoffman Ra, Harris SD. Spontaneous cerebrospinal fluid leaks originating from multiple skull base defects. Skull Base Surg 1996; 6(4): 227-30.

9. Wind JJ, Caputy AJ, Roberti F. Spontaneous encephaloceles of the temporal lobe. Neurosurg Focus 2008; 25(6): 11.

10. Kristin J, Betz CS, Stelter K, Berghaus A, Leunig A. Frontal sinus obliteration: a successful treatment option in patients with endoscopically inaccessible frontal mucoceles. Rhinology 2008; 46(1): 70-4.

11. Lopatin AS, Kapitanov DN, Potapov AA. Endonasal endoscopic repair of spontaneous cerebrospinal fluid leaks. Arch Otolaryngol Head Neck Surg 2003; 129 (8): 859-63.

12. Cavallo lM, Messina A, Cappabianca $P$ et al. Endoscopic endonasal surgery of the midline skull base: Anatomical study and clinical considerations. Neurosurg Focus 2005; 19(1): E2.

13. Becker SS, Duncavage JA, Russel PT. Endoscopic endonasal repair of difficult-to-access cerebrospinal fluid leaks of the frontal sinus. Am J Rhinol Allergy 2009; 23(2): 181-4
14. Petruzzelli GJ, Stankiewicz JA. Frontal sinus obliteration with hydroxyapatite cement. Laryngoscope 2002; 112(1): 32-6.

15. Taghizadeh F, Krömer $A$, Laedrach K. Evaluation of hydroxyapatite cement for frontal sinus obliteration after mucocele resection. Arch Facial Plast Surg 2006; 8(6): 416-22.

16. Mathur KK, Tatum SA, Kellman RM. Carbonated apatite and hydroxyapatite cement in craniofacial reconstruction. Arch Facial Plast Surg 2003; 5: 379-83.

17. Wormald PJ, Ananda A, Nair S. Modified endoscopic Lothrop as salvage for the failed osteoplastic flap with obliteration. Laryngoscope 2003; 113(11): 1988-92.

18. Gross WE, Gross CW, Becker D, Moore D, PHILLIPS D. Modified transnasal endoscopic Lothrop procedure as an alternative to frontal sinus obliteration. Otolaryngol Head Neck Surg 1995; 113 (4): 427-34.

19. WORMALD PJ. Salvage frontal sinus surgery: The endoscopic modified Lothrop procedure. Laryngoscope 2003; 113(2): 276-83.

20. Ulualp SO, Carlson TK, Toohill RJ. Osteoplastic flap versus modified endoscopic Lothrop procedure in patients with frontal sinus disease. Am J Rhinol 2000; 14(1): 21-6. 